\title{
Breast angiosarcoma in a young patient, case report
} Victor E Valdespino ${ }^{*}$, Israel Lopez Matamoros ${ }^{2}$, Maricruz Rivera Hernandez ${ }^{2}$, German Maytorena Cordova ${ }^{2}$ Argelia Camarillo Quesada $^{2}$
and Victor Valdespino Gomez

${ }^{1}$ General Hospital, Campeche, Mexico

${ }^{2}$ Luis Castelazo Ayala Hospital, Mexico City, Mexico

${ }^{3}$ National Academy Surgery, Mexico City, Mexico

\begin{abstract}
We show a case report on primary breast angiosarcoma, clinical presentation, histopathology findings, and a brief revision about breast angiosarcoma.

The patient develops a rapidly grown breast tumor, without diagnosis until she arrived at oncology gynecology services. She is 22 years old, this kind of angiosarcoma is not relational to radiotherapy or family cancer syndrome.

Ower group performed a simple mastectomy plus major pectoral resection, no axillary dissection was necessary and chemotherapy treatment.

The patient has 5 months of free period disease without relapse symptoms.

Ultrasound or mammography is not much useful in diagnosis about it. Magnetic resonance is more helpful in non-epithelial breast tumors.

Free border resection, the grade of the tumor, and suspect to the aggressive tumor are strong points to consider in this kind of breast tumor. Chemotherapy until now is not beneficial in adjuvant treatment just palliative election. Survival media time is $35-40$ months after treatment.
\end{abstract}

\section{Introduction}

Angiosarcomas of the breast is an infrequent clinical condition, accounting for $0.04 \%$ in all breast cancers. Luini in Milan estimated an incidence between 0.002 to 0.005 per year. Breast angiosarcomas are divided into two categories, primary and secondary (radiationassociated) [1].

Those tumors share similar histological features, clinical appearance is alike, but with a radiotherapy and surgery precedent, and the prognosis is bad in both cases. The clinical presentation in primary angiosarcoma is two decades or three before than secondary angiosarcoma $[2,3]$.

There is the association between primary angiosarcoma and onset pregnancy but do not is solid this affirmation maybe is a coincidence in the age group and some authors are reported primary angiosarcoma men [4].

Primary angiosarcoma is a malignant vascular neoplasm that arises within the breast parenchyma, while secondary angiosarcoma usually arises in the cutaneous tissue and might invade the breast parenchyma secondarily. In most series, patients with secondary angiosarcoma had significantly shorter survival time than those who had primary angiosarcoma and were more prone to local and distant recurrence. Ming and coworkers reported that results of a clinical trial suggested that secondary breast angiosarcoma had more aggressive tumor phenotype than primary angiosarcoma at presentation [5].

\section{Clinical case}

The patient is a 22 years old healthy female, in December 2019 began with pain and increased volume in the right breast; after that she takes an appointment with a general gynecologist and prescribes him, topic progestagen cream without response. The tumor was continuous growing on breast and pain, then get oncology gynecology by an ower group and we performed a cut needle biopsy with report primary breast angiosarcoma.

A total mastectomy with pectoralis major muscle was performed without complications and she was discharged from hospital 2 days after surgery.

The pathological final report is breast angiosarcoma high grade, 10 $\mathrm{cm}$ major diameter, all edges were tumor negatives more than $2 \mathrm{~cm}$. No axillary dissection was performed.

We used to scan tomography because obtain magnetic resonance in some hospitals in Mexico is difficult.

The patient received radiotherapy $50 \mathrm{~Gy}$ in a field breast with toxicity grade I - II without complications.

After 5 months of free period disease, she does not have signals of local recurrence or axillar or distant metastasis however the patient has relapse risk distant or local.

We present a case report and a review of breast angiosarcoma. Angiosarcoma breast is reported from 17 to 84 years old in a female patient the present case is on a very young patient, this disease is very infrequent in young persons and de clinical presentations do not have

${ }^{\star}$ Correspondence to: Victor E Valdespino, General Hospital, Campeche, Mexico, E-mail: edvaldespinocg@yahoo.com

Key words: breast angiosarcoma, primary angiosarcoma, breast rare tumors

Received: June 06, 2020; Accepted: June 26, 2020; Published: June 29, 2020 
risk factors in primary breast angiosarcoma. She does not report history about family cancer.

\section{Discussion}

Primary sarcomas of the breast are an infrequent clinical condition, they are an incidence of fewer than $1 \%$ of all breast cancers. Sarcomas arise from mesenchymal tissue of the mammary gland, one of the most common histological subtypes of primary sarcoma of the breast is angiosarcoma. There is primary angiosarcoma this tumor develops during the third or fourth decades of life. And the secondary breast angiosarcoma, radiation-induced soft tissue sarcoma, after conservative surgical treatment and radiotherapy on the breast.

Adem and his team found that angiosarcoma and fibrosarcoma in the breast are most frequent breast sarcomas, and the size tumor is the most important survival prognostic factor, and they do not launch axillar nodal metastasis [6].

Primary angiosarcoma of the breast typically presents as a palpable breast mass in women in the third and fourth decade of life. Secondary angiosarcomas of the breast are generally found in older women following breast cancer treatment. Secondary angiosarcoma may present with skin lesions such as nodules or discoloration, due to damage of lymphatic or vascular channels following surgery and irradiation. There is typically a latency period of 10- 20 years following breast conservation and radiotherapy [7]

There are Angiosarcoma Project (ASC project), an initiative enabling US and Canadian patients to remotely share their clinical information and biospecimens for research.

There are Angiosarcoma Project (ASC project) an initiative from the US and Canada, it can help to share remotely clinical information about angiosarcoma for current or futures research on this topic. (https://ascproject.org) [8].

This group found PIK3CA mutated genes for angiosarcoma has taken more importance; this topic in particular for breast angiosarcoma, they found the response of pembrolixumab in those kinds of patients even complete responses in two patients in metastatic disease [8].

Targeted inhibition of the PIK3, signaling pathway can have deep effects on cancer cell growth through numerous mechanisms [9].

How is the best target the PIK3 pathway in cancer treatment remains a topic of active investigation in particular for breast angiosarcoma?

Relapse metastatic angiosarcoma for a medical oncologist is a serious challenge, standard chemotherapy nor targeted agents has demonstrated good and durable responses. Drugs used for metastatic angiosarcoma include doxorubicin, paclitaxel, docetaxel, and bevacizumab.

Small case series have documented durable benefits of metronomic chemotherapy in relapsing angiosarcoma [10].

Sher and coworkers reported than adjuvant chemotherapy using anthracycline and ifosfamide or gemcitabine and a taxane did no significantly improve recurrence-free survival compared with patients who did not receive chemotherapy treatment [11]. However, the administration of palliative chemotherapy resulted in a $48 \%$ response rate. In the case of secondary angiosarcomas induced by radiation treatment docetaxel showed promise response [12].

In other small series of Biswas and workers reported a median overall survival was 37.4 months (range, 8.7-92.8 months). The median RFS was 17.9 months (range, 2.5- 69.4 months). They used to as chemotherapy paclitaxel and gemcitabine like palliative treatment.

In addition to it, Four of 5 patients had negative mammograms [13].

In Sri Lanka reported a bilateral synchronic angiosarcoma after surgery and radiotherapy (secondary breast angiosarcoma) in a patient of 62 years old, she develops those tumors with only 5 years of history to radiotherapy, the difference with frequent radio induced tumor between 10 or 20 years [14].

Ragavan in Singapore and coworkers give treatment to 13 patients with angiosarcoma, 5 patients received any axillar surgical treatment, and they do not detect axillary nodal metastasis and neither axillar relapse. Recurrence was usual to the bone, liver, ovary, contralateral breast, peritoneum [15].

Breast angiosarcoma has limited available literature with small and inconclusive results, but surgery treatment with wide and clear tumor border is the best treatment, tumor size and grade are principal prognostic factors (like others sarcomas) adjuvant treatment is unclear in results, axillar nodal treatment is not necessary, molecular trigger therapy someday will be a useful treatment in the metastasic stage.

The tumor cells from breast angiosarcoma proved to be immunoreactive to vascular markers (CD31 and CD34) and negative to epithelial markers; is helpful to confirm breast angiosarcoma but is very important suspect it with the clinical appearance [16].

However, we do not use immunochemistry in the present case. Mammographic and sonographic findings are non-specific and can be normal in up to a third of cases. Sonographic findings are non-specific, and the mass can be hyperechoic, hypoechoic, or heterogeneous. 7 Heterogeneous sonographic appearances may be due to hemorrhage of the tumor into the surrounding stroma. In secondary angiosarcoma, dermal lesions may be difficult to distinguish from post-irradiation skin thickening, skin thickening could be de only stage found in ultrasound [17].

In primary angiosarcoma, a heterogeneous mass is demonstrated on MR imaging with low signal intensity on $\mathrm{T} 1$ images and high signal on T2 images. On dynamic phase imaging, kinetic enhancement depends on tumor grade. High-grade tumors may demonstrate rapid enhancement with washout kinetics, but lower-grade tumors usually demonstrate plateau or persistent enhancement kinetics [18] (Figures 1-6).

\section{Conclusions}

Breast angiosarcoma is an aggressive tumor with poor survival, five rear overall rates range $38-78 \%$, and 5 years free survival between 38

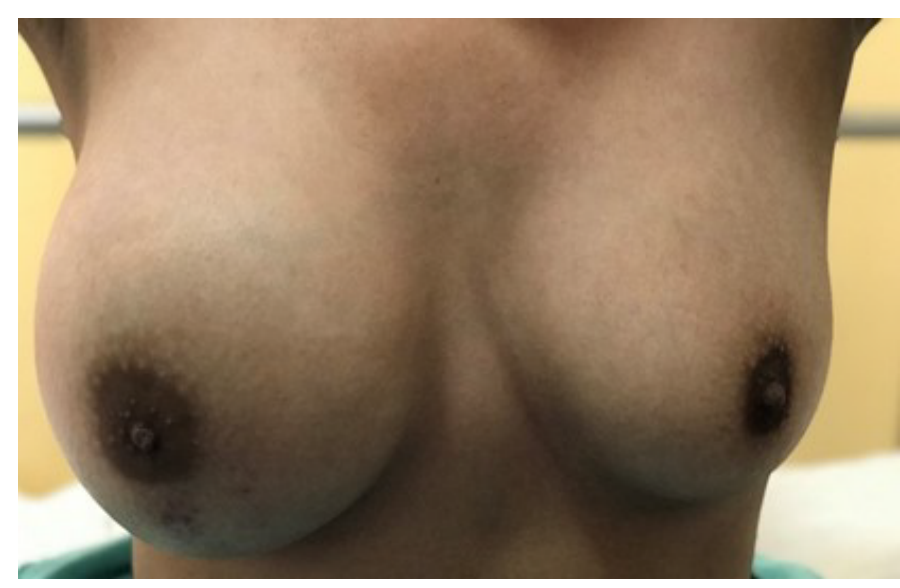

Figure 1. Clinical photography - asymmetric breast 


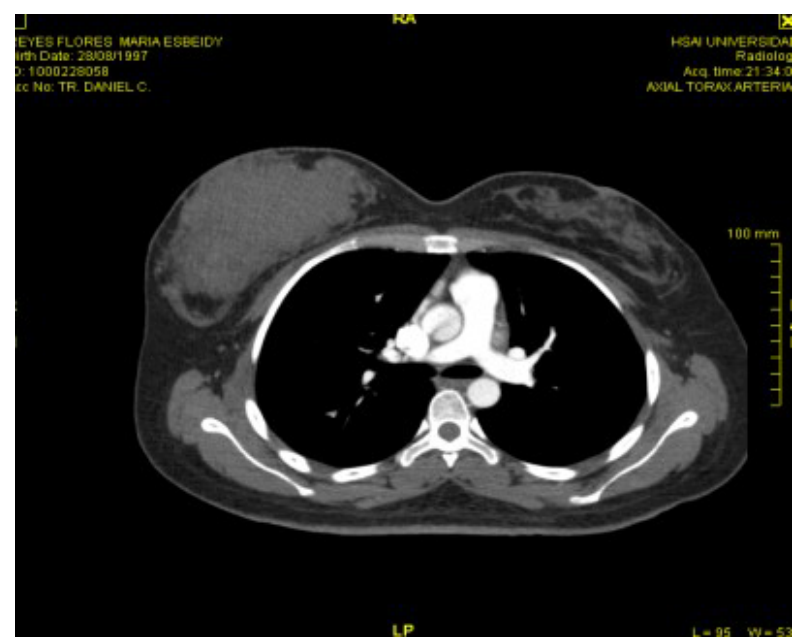

Figure 2. Chest Tomography of breast tumor

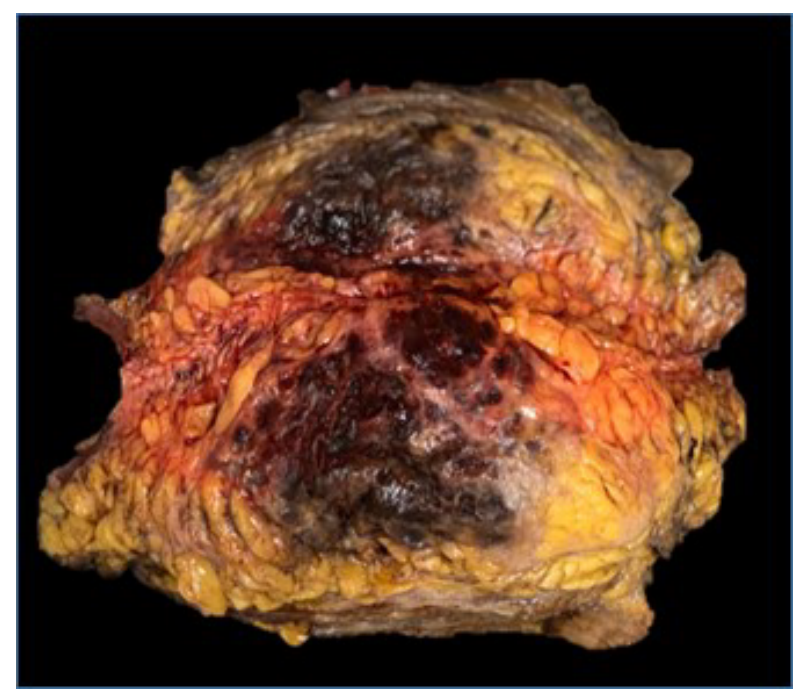

Figure 3. Whole breast tumor with ink

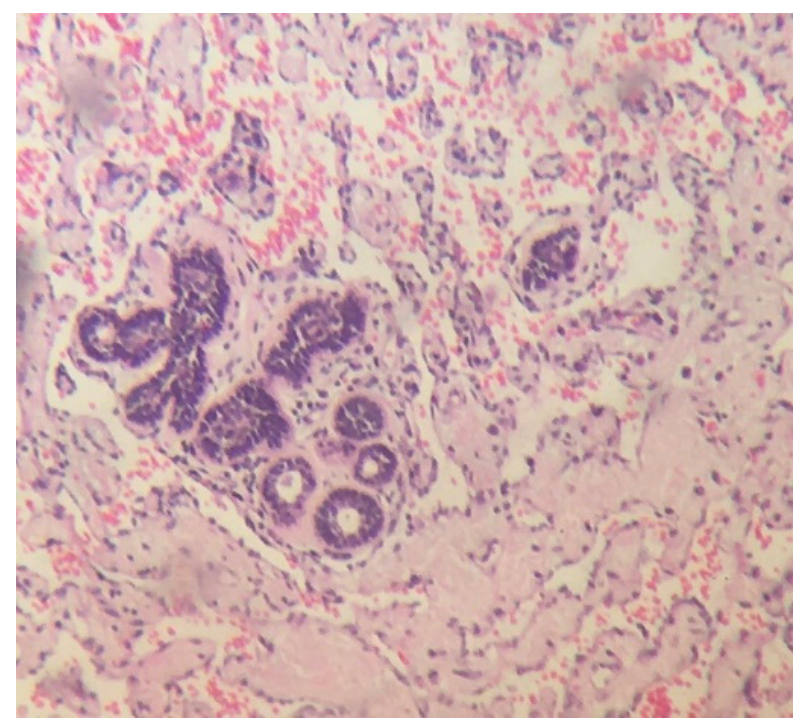

Figure 4. Lobular breast tissue surrounded by several vascular canal anastomosis (microphotograph)

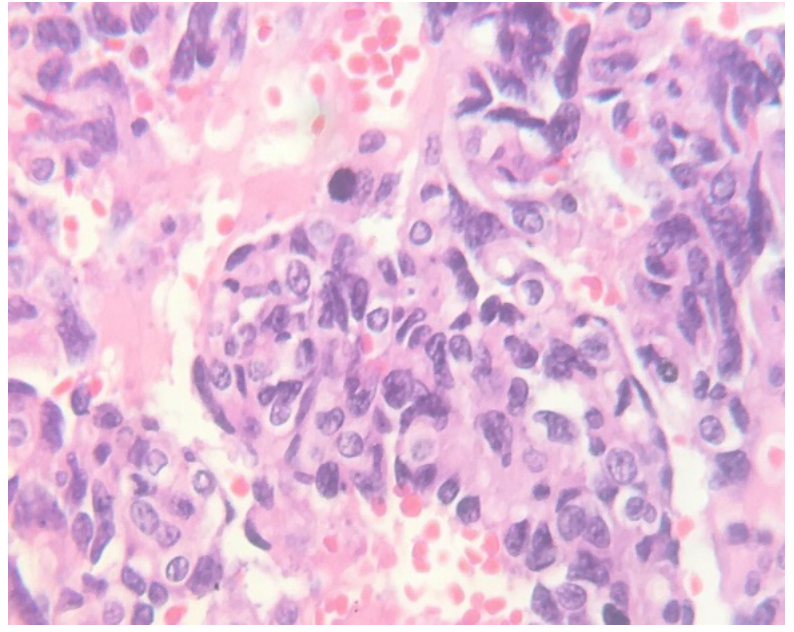

Figure 5. Tumor solid form with a vascular branch growing and mitotic activity

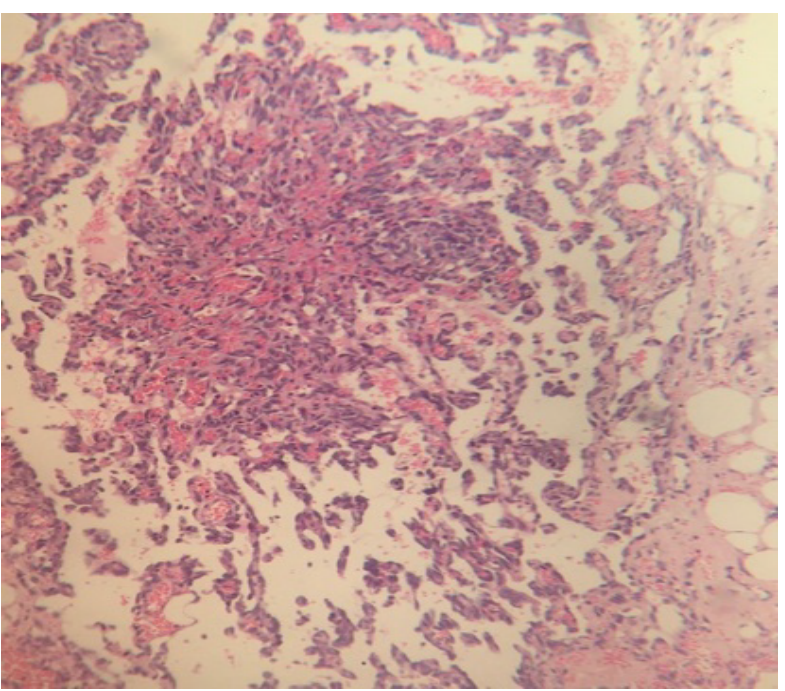

Figure 6. Microphotograph show intravascular papillar growing

and $56 \%$. Size tumor looks to be the most important factor in survival, (less than $5 \mathrm{~cm}$ ). Early diagnosis with correct (with R0 or negative margins ) surgical treatment can give patients a good quality of life, but in a rare tumor we need more reports also clinical essays if we want to improve the life of these patients. Axilar nodal dissection is not helpful for this tumor.

As well as advanced tumor grade (grade 3) is associated with significantly worse overall survival.

Adjuvant treatment is not much useful for radiotherapy or chemotherapy. The early clinical suspect is the key and does not delay treatment until now is the correct behavior.

\section{References}

1. Luini A, Gatti G, Diaz J, Botteri E, Oliveira E, et al. (2007) Angiosarcoma of the breast: the experience of the European Institute of Oncology and a review of the literature. Breast Cancer Res Treat 105: 81-85.

2. Brodie C, Provenzano E (2007) Vascular proliferation of the breast. Histopathology 52: $30-44$.

3. Rosen PP (2001) Rosen's breast pathology, 2nd eds. Philadelphia: Lippincott Williams and Wilkins. 
4. Bernathova M, Jaschke W, Pechlahner C, Zelger B, Boner G (2006) Primary angiosarcoma of the breast associated Kasabach-Merritt syndrome during pregnancy. The Breast 15: 255-258.

5. Yin M, Wang W, Drabick JJ, Harold HA (2017) Prognosis and treatment of nonmetastatic primary and secondary breast angiosarcoma: a comparative study. BMC Cancer 17: 295

6. Adem C, Reynolds C, Ingle JN, Nascimento AG (2004) Primary breast sarcoma: Clinicopathologic series from the Mayo Clinic and review of the literature. Br J Cancer 91: 237-241.

7. Kong J, Shahait AD, Choi L (2020) Radiation-induced undifferentiated pleomorphic sarcoma of the breast. BMJ Case Rep 13: e232616.

8. Painter CA, Jain E, Tomson BN, Dunphy M, Stoddard RE, et al. (2020) The Angiosarcoma Project: enabling genomic and clinical discoveries in rare cancer through patient-partnered research. Nat Med 26: 181-187.

9. Gelmann EP, Sawyers CL, Rauscher FJ, ed. (2014) Molecular Oncology. Cambridge University Press.

10. Pramanik R, Gogia A, Malik PS, Gogi R (2017) Metastatic primary angiosarcoma of the breast: Can we tame it the metronomic way. Indian J Med Paediatr Oncol 38: 228-231.

11. Sher T, Hennessy BT, Valero V, Broglio K, Woodward WA, et al. (2007) Primary angiosarcomas of the breast. Cancer 110: 173-178.
12. Mano MS, Fraser G, Kerr J, Gray M, Evans V, et al. (2006) Radiation-induced angiosarcoma of the breast shows a major response to docetaxel after the failure of anthracycline-based chemotherapy. Breast 15: 117-118.

13. Biswas T, Tang P, Muhs A, Ling M (2009) Angiosarcoma of the breast. Am J Clin Oncol 32: 582-586.

14. Jayarajah U, Nagodavithane K, Basnayake O, Seneviratne S (2020) Unusual Presentation of bilateral radiation-induced angiosarcoma of the breast. Case Rep Oncol Med 2020: 5768438

15. Ragavan S, Lim HJ, Tan WS, Hendrikson J, Yongsheng CJ, et al. (2020) Axillary lymph node dissection in angiosarcomas of the breast: An Asian Institutional Perspective. Sarcoma 2020: 4890803.

16. Armegot CM, Roca EM, Quecedo EE, Gimeno CE (2012) Angiosarcoma of the skin after Breas Cancer Radiotherapy. Actas Dermosifiliogr 103: 55755-55759.

17. Glazebrook KN, Magut MJ, Reynolds C (2008) Angiosarcoma of the breast. AJR 190 533-538.

18. Neill A, D'Arcy C, McDermott E, O’Doherty A, Quinn C, et al. (2014) Magnetic resonance imaging appearances in primary and secondary angiosarcoma of the breast. J Med Imaging Radiat Oncol 58: 208-212.

Copyright: $(2020$ Valdespino VE. This is an open-access article distributed under the terms of the Creative Commons Attribution License, which permits unrestricted use, distribution, and reproduction in any medium, provided the original author and source are credited. 